\title{
Denosumab For The Treatment Of Bisphosphonate-Refractory Hypercalcemia of Malignancy - A Case Report
}

\author{
Vishal V Ramteke, M.D., ${ }^{1}$ Sudhir R Dash, M.D., ${ }^{2}$ Shubhadeep Bose, M.D., ${ }^{3}$ Ritu Jain, M.D., \\ 'Registrar, Department of Nephrology, ${ }^{2}$ Consultant Nephrologist, \\ ${ }^{3}$ Registrar, Department of Oncology, ${ }^{4}$ Consultant Oncologist \\ Jaslok Hospital and Research Centre, 15-G Deshmukh Marg, Mumbai, India- 400026
}

\section{A B S T RACT}

Hypercalcemia of malignancy caused primarily by tumor-induced bone resorption may lead to renal failure, coma, and death. Patients treated with routine intravenous bisphosphonates may not respond or may relapse on therapy. Denosumab, a monoclonal human antibody which binds to RANKL, inhibits the osteoclast mediated bone resorption and hypercalcemia. We report a case of hypercalcemia in patient with metastatic carcinoma breast refractory to bisphosphonate therapy responding to denosumab and to the best of our knowledge is the first reported case in Indian literature. JMS 2015; 18(2):153-155

Keywords - Denosumab, Hypercalcemia of malignancy (HCM), RANKL, Bisphosphonates

\section{INTRODUCTION}

Hypercalcemia of malignancy (HCM) is a life threatening complication seen in advanced cancer which if untreated can lead to renal failure, progressive mental impairment, coma and death. Mechanisms are osteolytic resorption near the malignant cell invasion and parathyroid hormone related peptide (PTHrP) induced increased bone resorption and renal calcium retention. ${ }^{1}$ Conventionally HCM is treated with combination of hydration, diuresis with loop diuretics, calcitonin, steroids and bisphosphonates. However in many cases, HCM may be refractory or get relapsed on intravenous bisphosphonate therapy. Recurrent use of bisphosphonates carries a risk of kidney injury and osteonecrosis of jaw. In those patients, use of denosumab- a fully human monoclonal antibody which binds RANKL thereby reducing the osteoclastic bone resorption has shown good response. We report a case of hypercalcemia in patient with metastatic carcinoma breast refractory to bisphosphonate which responded well to Denosumab.

\section{Correspondence}

Dr. Vishal V Ramteke, M.D.

Department of Nephrology, Jaslok Hospital and

Research Centre, 15-G Deshmukh Marg,

Mumbai, India- 400026

Email:vvramteke@gmail.com

\section{CASE REPORT}

A 61 year old female with a history of carcinoma breast with metastasis to lung, liver and peritoneum presented with confusion and dehydration to the oncology services. She had undergone modified radical mastectomy two years back followed by chemo-radiotherapy. On evaluation she had no focal neuro-deficit and MRI brain done to rule out CNS mets was normal. The albumin corrected serum calcium level (ACSC) was $15.8 \mathrm{mg} / \mathrm{dl}$ with serum PTH - $34.4 \mathrm{pg} / \mathrm{ml}$ and vitamin D3 $42.3 \mathrm{ng} / \mathrm{ml}$ which were within normal limits. In past 6 months she was admitted multiple times with persistent hypercalcemia which was treated with injection Zolendronic acid, Calcitonin, Hydrocortisone and saline diuresis, lastest two weeks ago. Since the hypercalcemia was refractory to Zolendronic acid, nephrology consult was sought for further management.

After evaluation by nephrology services, the hypercalcemia was attributed to humoral hypercalcemia of malignancy, though PTH-rP levels were not done due to non-availability in the region. A trial of inj Calcitonin, Hydrocortisone and saline diuresis was also tried. As there was no clinical response after two days and ACSC still $>15 \mathrm{mg} / \mathrm{dl}$, it was decided to use inj Denosumab, a human monoclonal 
antibody binding RANKL. Denosumab is being used offlabel by few centres worldwide to treat the bisphosphonaterefractory hypercalcemia though initially approved by FDA for the prevention of skeletal-related events in metastatic solid tumors. Inj Denosumab $120 \mathrm{mg}$ s.c was given on day 1 , 8, 15, 29 and 60. After the initiation of denosumab, the calcium level of the patient fell rapidly to near normal levels on 7 day with clinical improvement in sensorium and oral intake. The patient was continued on tab Cinacalcet $30 \mathrm{mg}$ $\mathrm{q} 12 \mathrm{~h}$ as a denosumab sparing strategy due to prohibitive cost. Tab Cinacalcet was subsequently tapered and stopped with normalisation of ACSC. The response achieved is still maintained on 3 months of follow up. (Table 1, Figure 1)

\section{DISCUSSION}

Denosumabis a novel, fully human monoclonal antibody, which prevents the Receptor Activator of Nuclear factor Kappa B Ligand (RANKL) from binding to its receptor and inhibits osteoclast development, activation, and survival. ${ }^{2}$ By decreasing the osteoclast mediated bone resorption it thus reduces the consequent hypercalcemia. It was initially approved by US FDA in 2010 for prevention of skeletalrelated events in metastatic solid tumors. In phase III studies, it was noted that there was 52\% lower incidence of HCM, more pronounced suppression of bone-turn given. Tab Cinacalcet $30 \mathrm{mg}$ q12h was over markers and significant hypocalcemia with denosumab than with

Table 1: Shows levels of ACSC and ionised calcium as per the day after initiation of injection Denosumab

\begin{tabular}{|l|c|c|c|c|c|c|}
\hline & DAY 0 & DAY 3 & DAY 7 & DAY 30 & DAY 60 & DAY 90 \\
\hline $\begin{array}{l}\text { Albumin corrected serum } \\
\text { calcium (ACSC) }\end{array}$ & 15.9 & 13.1 & 11.8 & 10.6 & 9.4 & 8.2 \\
\hline lonized Calcium & 9.9 & 7.8 & 6.2 & 5.4 & 4.8 & 4.2 \\
\hline
\end{tabular}

Figure 1: Shows the response curve in ACSC and ionised calcium according to the day after initiation of inj Denosumab

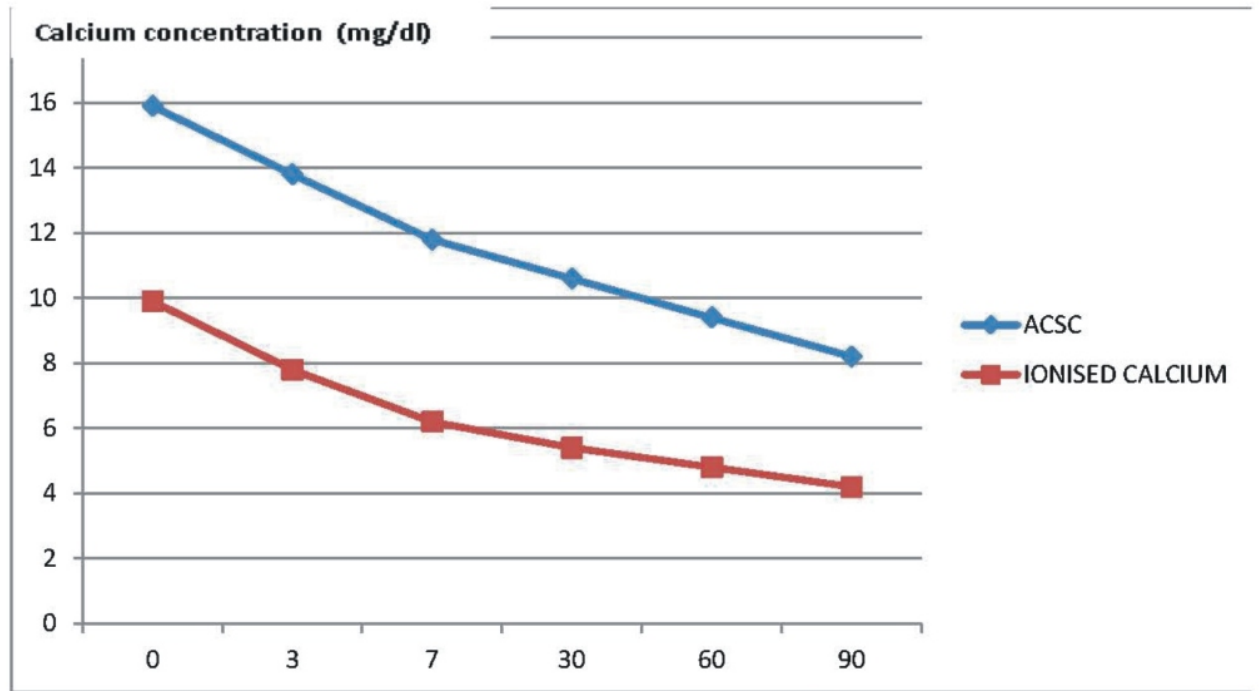

Days after inj Denosumab 
zoledronic acid ${ }^{3}$. This observation has prompted its off label use for HCM refractory bisphosphonate therapy with few successful cases reported in literature. ${ }^{4-6}$

There has been dearth of structured clinical trials studying the use of denosumab for refractory hypercalcemia. In study by Mimi I. Huet al, ${ }^{7}$ patients with advanced cancer and persistent hypercalcemia after incomplete response or relapse after recent bisphosphonate treatment, denosumab lowered serum calcium to grade 1 or lower $(\leq 11.5 \mathrm{mg} / \mathrm{dL})$ in $80 \%$ of patients by 10th day and the response was maintained for a median of 26 days. The dose of Inj Denosumab $120 \mathrm{mg}$ s.c was given on day 1, 8, 15, 29 and then every 4 weeks. Similar response was noted in our patient too. Hypocalcemia, hypophosphatemia, osteonecrosis of jaw and hypersensitivity reaction may be noted with Denosumab. It doesn't require renal dose adjustment. There is recent evidence that cinacalcet, an allosteric modulator of the calcium- sensing receptor $(\mathrm{CaR})$ attenuates PTHrP-mediated elevations in blood ionized calcium by increasing calcitonin release. ${ }^{8,9}$ Thus denosumab as induction agent and cinacalcet as maintenance are novel treatment options in such difficult cases.

In conclusion, we suggest the use of denosumab in combination with cinacalcet as a successful treatment option for bisphosphonate refractory hypercalcemia of malignancy. Prohibitive cost and lack of validated data about its efficacy are the issues need to be addressed before its widespread use. To our knowledge, this is the first reported case in India of management of malignant hypercalcemia in a solid tumor with denosumab.

\section{REFERENCES}

1. Stewart AF. Clinical practice. Hypercalcemia associated with cancer. N Engl J Med. 2005; 352 (4): 373-379.

2. Baron R, Ferrari S, Russell RG: Denosumab and biphosponates: Different mechanisms of action and effects. Bone 48:677-692, 2011
3. Martin $\mathrm{M}$, Bell $\mathrm{R}$, Bourgeois $\mathrm{H}$, et al. Bonerelatedcomplications and quality of life in advanced breast cancer: results from a randomized phase III trial of denosumab versus zoledronic acid. Clin Cancer Res.2012;18(17):4841-4849.

4. Sosipatros A. Boikos, Hans-Joerg Hammers. Denosumab for the Treatment of BisphosphonateRefractory Hypercalcemia; Journal of Clinical Oncology, Vol 30, No 29 (October 10), 2012: 299

5. Amanda Dietzek, Kelly Connelly, Michael Cotugno,Sylvia Bartel, Anne M McDonnell : Denosumab in hypercalcemia of malignancy: A case series- J Oncol Pharm Pract Jan 10, 2014:1078155213518361

6. Jason Adhikaree, Yvette Newby, Santhanam Sundar: Denosumab should be the treatment of choice for bisphosphonate refractory hypercalcaemia of malignancy - BMJ Case Reports 2014; doi:10.1136/bcr-2013-202861

7. Mimi I. Hu, Ilya Glezerman, Sophie Loboulleux, Karl Insogna, Rasim Gucalp, Waldemar Misiorowski, Bennett Yu, Wendy Ying, Rajul K. Jain- Denosumab for Patients With Persistentor Relapsed Hypercalcemia of Malignancy Despite Recent Bisphosphonate Treatment- J Natl Cancer Inst;2013;105:1417-1420

8. Anneke Bech, Koen Smolders, Darryl Telting, Hans de Boer Cinacalcet for Hypercalcemia Caused by Pulmonary Squamous Cell Carcinoma Producing Parathyroid Hormone- Related Peptide,Case Rep Oncol. 2012 Jan-Apr; 5(1): 18.

9. Colloton M, Shatzen E, Wiemann B, Starnes C, Scully S, Henley C, Martin D Cinacalcet attenuates hypercalcemia observed in mice bearing either Rice H500 Leydig cell or C26-DCT colon tumors - European journal of pharmacology 712:1-3 $2013 \mathrm{Jul} 15 \mathrm{pg}$ 8-15 